\title{
La compra de símbolos sociales frente al consumo de objetos
}

\author{
Vidal Díaz de Rada \\ Universidad Pública de Navarra. Departamento de Sociología \\ Campus de Arrosadía, s/n. 31006 Pamplona
}

\section{Resumen}

Este artículo analiza el comportamiento del consumidor prestando una atención especial a la compra de "símbolos sociales" y al significado del término necesidad en la sociedad actual, considerando que desde una perspectiva sociológica este concepto debe definirse teniendo en cuenta que "el volumen de las necesidades naturales y la manera de satisfacerlas es un producto que depende del nivel cultural de un país». Una vez analizados los elementos implícitos en el acto de compra - posesión del producto, utilidad del mismo, ritual de la compra - se analizará la incidencia que los hábitos de compra tienen en la estructuración de la vida cotidiana; empleando para ello las respuestas a una encuesta aplicada a una muestra representativa de la sociedad Navarra.

Palabras clave: consumo, comportamiento del consumidor, necesidad social, símbolos, proceso de apropiación, formas de consumo.

\section{Abstract. The buying of social symbols front object's consumption}

This article analyzes the consumer's behavior playing special attention to the buying of "social symbols" and to the meaning of the concept neccesity in the current society, considering that - from a sociological point of view - this concept must be defined taking into account that uthe volume of the natural neccesities and the way of satisfying them is a product which depends on the cultural level of the country". Once the elements involved in the act of buying have been analyzed (posession of the product, it's utility, the buying ritual), we will analyze how the buying habits influence on the structure of everyday's life, using for this purpose the answers given to a survey carried out to a representative sample of the navarran society.

Key words: consumption, consumer behavior, social need, symbols, appropiation, forms of consumption.

\section{Sumario}

1. "Expansión artificial» de la demanda en las sociedades actuales

2. Elementos subyacentes implícitos en el proceso de compra

3. Incidencia de los hábitos de compra en la estructuración de la vida cotidiana
4. Reflexión final

Anexo I: ficha técnica del estudio

Anexo II: preguntas utilizadas

Bibliografía 
El fin de este trabajo es realizar, desde una perspectiva crítica, un análisis de la compra de "símbolos sociales» frente al. consumo de objetos, adoptando como punto de partida una definición del concepto necesidad como elemento dinamizador de la conducta de compra del individuo. Según los estudiosos del comportamiento del consumidor (Alonso Rivas, 1984: 278-287), el descubrimiento de la necesidad hace aparecer en el individuo un estado de carencia que, concretado en el deseo de un objeto determinado, le conduce a la intención de adquirirlo. Éste descubrimiento de la necesidad surge por la percepción - generalmente determinada por situaciones sociales - de una diferencia entre el estado ideal y el estado actual o real. El estado real es la percepción de la situación actual, muy influenciada por circunstancias pasadas, mientras que el estado ideal es ese objetivo o esa posición que el individuo desea alcanzar en un tiempo futuro (Loudon y Della-Bitta, 1993: 483-502).

No obstante, en este artículo vamos a adoptar un objeto de estudio más amplio, puesto que nuestro ámbito de análisis no son las conductas individuales de compra, sino estudiar en qué medida la sociedad —en sí mismaactúa como generadora de necesidades. Desde esta perspectiva, el artículo comienza con una exposición del desarrollo de la sociedad capitalista y como ésta se ha visto obligada a transformar sus estructuras de producción y consumo a fin de eliminar las crisis de sobreproducción de sus comienzos.

Posteriormente se estudia la incidencia que los hábitos de compra tienen en la estructuración de la vida cotidiana, contemplando aspectos como el tiempo empleado viendo escaparates, la compra de objetos que no son utilizados y los motivos por los cuales se dejan de utilizar los productos adquiridos. El artículo finaliza con la reflexión general de los hallazgos encontrados a lo largo de todo el trabajo.

\section{1. "Expansión artificial» de la demanda en las sociedades actuales}

El sistema imperante en Occidente desde el siglo XVII hasta nuestros días ha sido el capitalismo, aunque desde sus primeros tiempos hasta el momento presente ha sufrido grandes transformaciones. Werner Sombart (Nussbaum, 1933) distingue tres etapas en el desarrollo del capitalismo: el capitalismo temprano, desde la mitad del siglo XIII hasta mediados del siglo XVIII; el capitalismo pleno, desde 1750 a 1914, y el capitalismo avanzado, situado históricamente en el período posterior a la Primera Guerra Mundial.

El capitalismo temprano se caracterizaba por un escaso desarrollo de la industria y la técnica, y por unas prácticas económicas influidas por las ideas y la cultura medieval. Actividades económicas como el crédito con elevados intereses (usura) o disminuir el precio para arruinar a la competencia eran prácticas con fuertes sanciones sociales. La sociedad y la economía existen para el hombre, y no el hombre para servir a éstas.

En el capitalismo pleno cambian sustancialmente las ideas tradicionales de las épocas anteriores y se introducen en las relaciones económicas los principios de la ganancia y el racionalismo económico. El hombre deja de ser la 


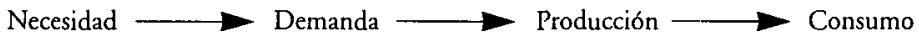

Figura 1. Dinámica de la producción en el siglo XIX. Fuente: J. Atalli y M. Guillaume, El antieconómico, Labor, Barcelona 1976, p. 215.

medida de todas las cosas y en su lugar emerge un poderoso sistema económico que impone como patrón de conducta el "conseguir el máximo beneficio económico". El "deber moral" del empresario es explotar al obrero para obtener de él un beneficio máximo. El mercado, a través de la oferta y la deman$\mathrm{da}$, se convierte en el regulador de toda la vida económica y está libre de los planteamientos restrictivos de épocas anteriores. Las ideas de Adam Smith sobre la existencia de una "mano invisible» que regula y distribuye la riqueza influyen poderosamente en toda la época (Smith, 1985).

En el siglo XIX la producción tiene como fin satisfacer las necesidades fundamentales del ser humano: la comida, el trabajo, la reproducción, etc. Las necesidades de las personas, a través de los procesos de demanda, ponen en marcha el sistema productivo creando objetos que serán consumidos para satisfacer sus necesidades (figura I).

Con el paso del tiempo las llamadas "necesidades básicas" han quedado satisfechas (en términos generales y para una gran parte de la población de las sociedades desarrolladas) y ha sido necesario crear unas «nuevas necesidades». A finales de siglo XIX Thorstein Veblen pone de manifiesto que hay ciertos consumos que se convierten en signos de admiración social. Estos consumos no están relacionados con la satisfacción de necesidades básicas, sino con el simbolismo en términos de admiración social que manifiesta la posesión de ciertos objetos: Cuando están cubiertas las necesidades básicas es preciso dotar a los objetos de un significado que va más allá de la utilidad concreta para la que fueron creados, es preciso darles un significado social.

El capitalismo avanzado se caracteriza por un gran desarrollo de la técnica. El mayor uso de la máquina de vapor, el motor de combustión, la electricidad, etc. da lugar a una creciente sustitución del hombre por la máquina. En este siglo se produce un gran cambio en la concepción del mercado; mientras que en el siglo XIX la tendencia era ahorrar y realizar la compra cuando se tuviera el dinero, en el siglo XX el proceso se invierte (Baudrillard, 1988: 180); primero se compra el producto y después se ahorra para pagar el préstamo ${ }^{1}$.

1. Un estudio realizado por la revista Eroski en 1990 indicaba que el $36 \%$ de las familias vasconavarras estaban endeudadas: Concretamente «el $21 \%$ de los hogares vascos deben mensualmente el $25 \%$ de sus ingresos, un $8 \%$ tiene cautivo entre el 25 y $40 \%$, y cerca del $2 \%$ de las familias (porcentaje que en Pamplona llega al 3,3\%) deberá pagar más del 60\% de sus ingresos mensuales a entidades financieras por el pago de préstamos, créditos, etc.». Fuente: Revista Eroski, 1990. 


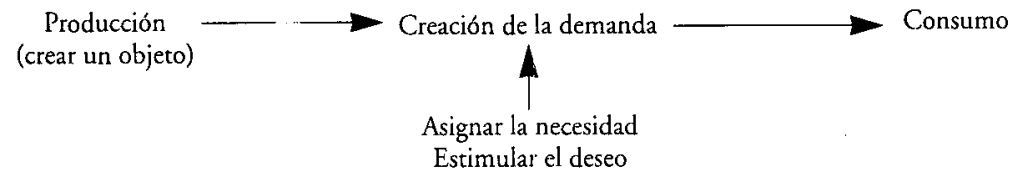

Figura 2. Dinámica de la producción en el siglo XX. Fuente: J.M. Pérez Tornero, La seducción de la opulencia, Paidós, Buenos Aires 1992, p. 19.

En este siglo, el crecimiento del consumo se convirtió en la condición necesaria para el incremento de la producción, de modo que «la potenciación de la demanda y del consumo evitaron el estrangulamiento de una producción superior a las rentas generadas por ésta" (Piñuel, 1983: 77), tal y como es formulado por Luis Alonso cuando expone que la sociedad de consumo surge cuando el capitalismo no pudo encontrar nuevos espacios para expandirse y tuvo que cambiar el antiguo modo de vida a fin de distribuir toda su producción (Alonso, 1985: 11-19). Una aplicación concreta de estas proposiciones puede extraerse de las memorias de un alto ejecutivo de la empresa de automóviles Ford (Iaccoca, 1985: 194) cuando afirma que cuando Henry Ford elevó en 1914 el salario de sus obreros de 2 a 5 dólares ("Five Dollars Day») no fue un gesto de bondad para mejorar sus condiciones de trabajo, sino para posibilitar que sus empleados pudieran adquirir sus coches. En otras palabras, Henry Ford fue uno de los creadores de la clase media americana: «Nuestro propio éxito depende, en parte, de lo que paguemos. Si repartimos mucho dinero ese dinero se gasta. Enriquecemos a los comerciantes, a los detallistas, a los fabricantes y a los trabajadores de todo tipo, y esta prosperidad se traduce en un incremento de la demanda de nuestros automóviles» (H. Ford I).

Esta realidad viene a revelar que el sistema económico actual está basado en el principio de la producción y el consumo en masa. Este sistema estimula constantemente a "desear" y comprar los objetos antes de tener el dinero necesario. La publicidad y la moda, junto con los patrones de comportamiento difundidos por los medios de comunicación estimulan (u obligan) al individuo a tener nuevas necesidades y, como no, a satisfacerlas ${ }^{2}$. De este modo, es el propio sistema productivo el que "crea un objeto" y posteriormente le «asigna» una necesidad para satisfacer ${ }^{3}$. El esquema del siglo XIX (figura 1 ) se ha transformado en la figura 2.

2. Ilustraremos esta idea con unas citas de H. Ford recogidas por G. Friedman: "Es necesario adelantarse a las necesidades del público y no permanecer sentados, contentándose con satisfacer lo que se llama una demanda». "Ja demanda o necesidad depende de la producción, no es la producción la que viene a llenar una demanda, de modo que la producción crea demanda, es decir, promueve las necesidades. "La educación del público, a cargo de la publicidad, desarrolla la demanda». «Una vez saturado un campo de necesidades, es menester preparar otron. (Friedman, 1977: 131-135).

3. Hecho que lleva a muchos autores a considerar que el elemento definitorio del capitalismo actual es la "producción de consumidores", y este elemento es la causa de la pérdida de interés de estos autores hacia la producción de mercancías. 
Puede afirmarse, por tanto, que la sociedad actual se ha especializado en la creación de necesidades ${ }^{4}$. La creación y el estímulo del deseo de los "nuevos productos" son utilizados para mantener el sistema productivo. Sistema productivo que paradójicamente comenzó para servir al ser humano (para hacerle la vida más cómoda) y satisfacer sus necesidades, pero no para someterlo a sus dictámenes productivos como es la situación actual: "Durante miles de años la función de la economía fue brindar los elementos cotidianos necesarios para la vida, para la subsistencia. Pero ahora la economía se ha engranado con las exigencias de la cultura. En el "nuevo capitalismo" la función de la economía es más para estimular la demanda del placer y del juego en el campo del consumon (Bell, 1987: 81).

Este planteamiento obliga a dirigir el tema hacia el estudio de las necesidades pero considerando que el análisis de las necesidades en sentido abstracto no tiene sentido, ya que el objeto de estudio sólo pueden ser necesidades concretas insertas en una sociedad y un modo de vida determinado (Heller, 1978: $36,23,115)$. Las necesidades, si bien tienen un componente biológico ${ }^{5}$, se manifiestan socialmente en un medio sociohistórico determinado. Este planteamiento adoptan autores como Maurice Halbwachs cuando analiza la influencia de los factores sociales en el comportamiento del consumidor y considera que las necesidades actuales son obra de un tipo de sociedad, y solamente pueden analizarse y explicarse mediante el análisis de esa determinada formación social. Para Halwbachs el actual sistema capitalista ha revestido de gustos y contenidos simbólicos la satisfacción de las necesidades básicas (1970: 440). Adoptando una perspectiva más economicista, John Kenneth Galbraith llega a una conclusión análoga cuando sostiene que un incremento en la producción genera - a través del aumento de salarios- los ingresos que permiten adquirirlo, de modo que a medida que una sociedad se vuelve más opulenta las necesidades son creadas por el proceso que las satisface (1985: 185).

No obstante, el autor que más lejos ha llevado la crítica sobre la creación artificial de las necesidades es Jean Baudrillard. Desde su punto de vista, fenómenos como la moda y la obsolescencia anticipada de los bienes no pueden ser entendidos desde una concepción unidireccional y aséptica del concepto necesidad, sino como un fenómeno necesario para la supervivencia del sistema

4. Un ejemplo muy concreto y conocido puede encontrarse al analizar un programa que fue emirido por una de las televisiones privadas. El citado programa comenzó a "hablar" y a hacer publicidad de un producto ("Cacao Maravillao") que nadie conocía y que, de hecho, no existía. Cuando la marca alcanzó notoriedad y la gente comenzó a preguntar en las tiendas por el producto, se vendió la marca (únicamente la marca) a una empresa de chocolate por una elevada suma de dinero (aproximadamente 900 millones) (Montañés, diciembre de 1991). En ésta misma línea, Erik Clark (1988: 24) señala que en Estados Unidos hay dos compañías, "Fruit of the Loom» y «Stentson» que no fabrican nada, su única función es permitir la utilización de sus nombres a cambio del pago de royalties.

5. Aconsejamos la lectura de las páginas 14-16, 71-74 de la obra de Doyal y Gought (1994), y del apartado "Crítica de la versión naturalista del concepto de necesidad", dentro del trabajo de L.E. Alonso (1986: 26-31). 
productivo. Para Baudrillard sólo hay necesidades porque el sistema las necesita (1971: 81-82)

Desde otro punto de vista, Herbert Marcuse establece una diferenciación entre necesidades verdaderas y falsas. Falsas son "las que intereses particulares imponen al individuo su represión: las necesidades que perpetúan el esfuerzo, la agresividad, la miseria y la injusticia. Las únicas necesidades que inequívocamente pueden reclamar satisfacción son las vitales: alimento, vestido y habitación en el nivel de cultura que ésta alcance. La satisfacción de estas necesidades es el requisito para la realización de todas las necesidades» (1987: 35-36). Basado en esta clasificación, L.E. Aloriso critica la escasa operatividad de esta definición al diferenciar entre deseo y necesidad: Desde su punto de vista, «la producción para el deseo es característica del capitalismo avanzado [...] No obstante, mientras que los deseos tienen sus bases más o menos remotas, la necesidad es previa al deseo y al objeto simbólico que origina este deseon (1986: 29-30).

De estas aportaciones se desprende que el término necesidad, como concepto genérico, tiene a su vez una dimensión individual y una dimensión social, y sólo desde este punto de vista se puede comprender que conceptos como el desgaste y la obsolescencia de los productos «no puedan analizarse independientemente de la estructura social». Así, mientras el desgaste es definido como una pérdida de aspecto y una degradación objetiva de la calidad del servicio prestado, la obsolescencia es un desarreglo del valor de uso del artículo debido no a su utilización sino a la evolución de la sociedad (Ceron y Baillon, 1980: 20-40). En este sentido, Luis E. Alonso señala tres aspectos que deben considerarse en el análisis de la "retirada» prematura de los productos: por un lado un desgaste cualitativo fruto del deterioro físico del producto, el desgaste funcional asociado al surgimiento de un nuevo producto, y una obsolescencia psicológica fundamentada en la promoción publicitaria (1990: 59-80).

En un intento de superar estos planteamientos, George Katona rechaza la afirmación de que las necesidades son impuestas artificialmente al consumidor, puesto que nuestros rasgos personales, las necesidades y las capacidades heredadas o innatas se ven modificadas por la experiencia. "La conducta económica es conducta adquirida en el sentido de que se desarrolla y cambia con la experiencia [...] No tiene ningún sentido establecer distinciones entre necesidades, deseos o conductas adquiridos y no adquiridos espontáneamente» (1968: 64).

6. Sin embargo, el análisis de Baudrillard es notablemente más extenso que el realizado por otros autores, como queda patente cuando critica determinados planteamientos que afirman que la necesidad está condicionada socialmente fundamentalmente a través de la publicidad (Pérez Agote 1978: 91). Así, Baudrillard considera NO que cada producto produzca necesidad de él, sino que es el proceso de producción el que elabora una lógica de necesidades, de modo que «la necesidad no es jamás la necesidad de un determinado objeto sino la "necesidad" de diferencia social", de modo que nunca podrá quedar satisfecha esta necesidad (Pérez Agote, 1978: 90-92) 
Del mismo modo José Castillo critica las concepciones anteriores argumentando que la publicidad y los otros mecanismos de la sociedad de consumo no incitan al incremento desmesurado del consumo de una forma tan determinista como lo exponen algunos autores. Desde su punto de vista, las técnicas de persuasión comercial influyen muy superficialmente en los procesos motivacionales del consumidor: «Al descubrimiento de los objetos ensalzados por la publicidad el ciudadano medio hubiera tardado mucho en llegar [...] Las modernas técnicas de persuasión no tienen más poder que el de convencer para el consumo al ya convencido y quizás provocar un asomo de vacilación en el reacio" (Castillo, 1987: 30-31).

Para José Castillo la "libertad de elección del consumidor" no depende tanto de los mecanismos de creación de necesidades sino de la limitación de los consumidores al tener que elegir únicamente entre productos que les son dados y sobre los que no tienen ninguna capacidad de crítica. Así, umientras no se consiga un equilibrio en la esfera del mercado de los bienes de consumo la autonomía real del consumidor será escasa». En este sentido, la racionalidad del consumidor se ve especialmente mermada en personas desfavorecidas socialmente: los ancianos, los niños, gente sin estudios, etc.

Si las necesidades son creadas socialmente y junto a estas necesidades aparecen de inmediato productos que las satisfacen, la libertad del consumidor queda relegada a la elección entre los diferentes productos existentes. La idea del consumidor soberano ha desaparecido y en la actualidad emerge un consumidor influenciado por la publicidad, la moda y los medios de comunicación. Ha desaparecido la personalidad autodirigida (Riesman) y en su lugar emerge un individuo normalizado y heterodirigido cuyo máximo deseo es ser aceptado y admitido pero sin destacar en exceso: «La persona dirigida por los otros no piensa tan a menudo en su vida en términos de una carrera individualizada. No busca la fama sino el respeto y el afecto de un jurado de pares amorfo y cambiante, si bien contemporáneo [...] Este consumidor anhela ser guiado por los otros antes que deslumbrarlos con su despliegue" (Riesman, 1981: 175).

Por lo expuesto hasta el momento, esta concepción del término necesidad adquiere una importancia capital en las modernas sociedades industriales como elemento dinamizador de conductas de compra. No obstante, el análisis del proceso de "expansión» de las necesidades debe estudiarse teniendo en cuenta no sólo los símbolos sociales asociados a los objetos, sino considerando también las condiciones estructurales de la sociedad avanzada cristalizadas en la multiplicidad de los tipos de redes comerciales, la determinación de "tiempos de compra» como las rebajas, la compra de «marcas" que identifica socialmente al permitir a su portador la identificación con su grupo y la exclusión de otros grupos, etc. Dicho de otro modo, el proceso de compra - en sí mismo- debe ser contemplado como un elemento generador de satisfacciones. 


\section{Elementos subyacentes implícitos en el proceso de compra}

En esta línea, y una vez contemplados los mecanismos que incitan a los individuos a incrementar sus niveles de consumo, nos detendremos a analizar cuales son los factores y los elementos valorativos que "están detrás» de cada adquisición: A mediados del presente siglo, George Simmel ya estableció una diferencia entre la utilización del objeto y la posesión del mismo como elementos independientes: «La forma económica del valor oscila entre dos límites: de un lado el deseo del objeto, que se vincula al sentimiento de satisfacción, posesión y disfrute anticipado; del otro, el mismo disfrute que, en sentido estricto, no constituye acto económico alguno [...] De tal manera, todo objeto tiene dos funciones: una la de ser utilizado y otra la de ser poseído» (Simmel, 1977: 59).

Esta diferenciación está indicando que los objetos tienen un valor "que va más allá de la propia utilidad funcional para la que son creados", valor relacionado - por un lado- con la utilización del producto, y por otro con la posesión de ese símbolo social que —en sí mismo- transmite significados culturales? ${ }^{7}$.

No obstante, en la sociedad actual habría que definir un tercer elemento, a nuestro juicio fundamental, como es el propio acto de compra con todo el ritual que lleva implícito: visitar varias tiendas, ver escaparates, etc. En esta línea, diversos autores consideran que existe dentro de la cultura del consumo un conjunto de elementos de tradición festivo-carnavalesca (Hetherington, 1992: 83-98) al adoptar los lugares de la compra un componente lúdico: "Las Arcadas y los Grandes Almacenes, "mundos de sueńo" de la cultura del consumidor, fueron para Walter Benjamin materializaciones de la idea marxista sobre "el fetichismo de la mercancía" ". Los nuevos grandes almacenes y arcadas eran templos en los que los bienes se adoraban como fetiches» (Featherstone, 1991: 22).

Desde esta perspectiva los actuales centros comerciales, los grandes almacenes, hipermercados, etc. tienen mucho en común con las ferias y exposiciones de antaño. Incluso hoy en día las compras en determinadas fechas del año (p.e. la Navidad) gozan de un importante componente festivo. Todos estos elementos forman parte del componente lúdico-festivo implícito en el acto de

7. No nos detendremos en una explicación de estas categorías ni en exponer otras clasificaciones puesto que supondría desviar nuestro objeto de estudio, aunque no por ello debemos obviar la "lógica fundamental de los objetos" propuesta por J. Baudrillard (1971: 66): "Lógica funcional del valor de uso, lógica económica del valor de cambio, lógica del intercambio simbólico y lógica del valor/signo".

Por otro lado, y adoptando otra perspectiva, aconsejamos a los interesados la consulta del cuadrado semiótico de los valores de consumo propuesta por A. Semprini (1992: 101-196) y la clasificación de los objetos del hogar realizada por Silverstone (1994: 20-26), así como la categorización de los "criterios de decisión" de O’Shaughenessy (1989), fundamentalmente lo referente a los "criterios integradores y adaptativos" (p. 135-154). 
compra $^{8}$. Rob Shields considera que estos comportamientos de compra, al combinar actividades de consumo y de ocio, generan una revalorización del concepto de sí mismo y de las relaciones personales dentro de los grupos, al tiempo que modificarán, a largo plazo, la distribución y el uso de los espacios sociales urbanos (1992: 1-20). En esta línea, y desde la perspectiva de Kevin Hetherington, debe considerarse al proceso de consumo corno «algo más» que una mera actividad de compra, ya que en ocasiones adquiere un papel fundamental en el comportamiento grupal puesto que contribuye notablemente en el mantenimiento de la estabilidad del grupo, sobre todo en «épocas de desestabilidad social y cuando emergen nuevos estilos de vida» (1992: 97) ${ }^{9}$.

8. En un excelente trabajo publicado recientemente, R. Laermans (1994: 121-144) realiza un análisis de la transformación de los procesos de compra desde el siglo XVII hasta nuestros días: Esta autora destaca el protagonismo ejercido por los grandes almacenes surgidos a mediados del siglo XIX (Bon Marche, Macy's, Marshall Field, etc.) en la modificación de los hábitos de compra y en el impulso y configuración de un "placer en la compra", producido fundamentalmente por un cambio de "comprar» a «ir de compras»: «El gran almacén era más que un espacio de consumo, era una visión del consumo [...]. Ir de compras se convirtió en una aventura de los sentidos" (p. 132).

9. Todos estos elementos son sintetizados por D.B. Holt (1995: 1-17) en un cuadro de doble entrada que, desde su punto de vista, muestra los valores implícitos en los objetos de consumo. Este cuadro está dividido verticalmente según el propósito de la acción de consumo -consumo como un fin en sí mismo o con un fin superior-y horizontalmente atendiendo a la estructuración de la acción: Consumo como acción interna (personal) o como acción externa (pensando en los otros). El "cruce" de estos dos conceptos produce cuatro celdas, que son definidas por el autor como las cuatro metáforas del consumo:

- Consumo como experiencia: El consumo como experiencia son todas las reglas y procesos mediante los cuales una persona adquiere un determinado objeto. Vendrían a ser todas las convenciones, hábitos, estrategias y estilos de actuación.

- Consumo como integracion: El objeto poseído constituye la identidad del poseedor; la persona ES a través de lo que posee.

Estos dos elementos son el resultado de una estructura de la acción interna (personal), y un propósito de la acción como fin en sí mismo y como fin superior, respectivamente. Por otro lado, las dos siguientes son producto de una acción externa:

- El consumo como clasificación está señalando el poder simbólico que tienen los objetos de consumo para clasificar a sus poseedores dentro de determinados grupos sociales. Ciertos objetos nos permiten clasificar a una persona dentro de un estrato elevado, objetos que previsiblemente perderán esta capacidad de diferenciación cuando su consumo y posesión se popularice.

- Consumo como juego: El consumo como juego está sugiriendo la existencia de un "tiempo social» de la compra, un "tiempo social» en el cual el proceso de compra es contemplado como un pasatiempo, como un momento lúdico-festivo por todos los elementos placenteros unidos a la acción de comprar.

\begin{tabular}{clll}
\hline & & \multicolumn{2}{c}{ Propósito de la acción de consumo } \\
\cline { 3 - 4 } & & Fin en sí mismo & Fin superior \\
\hline $\begin{array}{c}\text { Estructura } \\
\text { de la } \\
\text { acción }\end{array}$ & $\begin{array}{l}\text { Acción } \\
\text { interna }\end{array}$ & Experiencia & Integración \\
\cline { 2 - 4 } & $\begin{array}{l}\text { Acción } \\
\text { externa }\end{array}$ & Juego & Clasificación \\
\hline
\end{tabular}




\section{Incidencia de los hábitos de compra en la estructuración de la vida cotidiana}

Una vez realizada esta exposición sobre el concepto necesidad y los valores implícitos en los objetos de consumo, interesa conocer en qué medida estas necesidades son «impuestas» socialmente a los individuos. Dicho de otro modo, se trata de separar el hecho circunstancial de la compra (transacción de un objeto por su valor monetario) y analizar cuantas actividades de su vida diaria tienen relación con la adquisición del producto, qué porcentaje de su tiempo lo dedica a actividades más o menos paralelas y relacionadas circunstancialmente con el acto de compra ${ }^{10}$.

El punto de partida es la concepción que la conducta de compra es consecuencia y está desencadenada por la aparición de una necesidad en el individuo, necesidad que hace surgir en el consumidor una búsqueda de información y una posterior toma de decisiones. Como ya ha sido expuesto anteriormente, este trabajo parte de la premisa que la necesidad de comprar - en la mayoría de los casos- viene motivada NO por una carencia biológica o física (Atalli, 1976: 467-491 y Sauerman, 1983), sino por una carencia creada y fundamentada socialmente (Ballestero, 1982: 29-62).

Desde este punto de vista, se plantea como hipótesis que la compra en si, el proceso de adquisición del objeto (con todo el ritual que lleva implícito: ver escaparates, visitar varias tiendas, pedir consejos a los amigos, etc.) es un "elemento" que reporta gran satisfacción al individuo y que en ocasiones le conduce a comprar productos que no necesita y que nunca llegará a utilizar. Esta hipótesis está en línea con la tesis de Amando de Miguel cuando afirma «El comprar puede ser un placer en sí mismo, por eso el tiempo de compra se amplía sin cesar" (De Miguel, 1990: 73).

Con este fin se han formulado varias preguntas que hacen referencia al tiempo invertido en la compra, a la diferencia entre la compra y la utilización de los productos, y al conocimiento de los motivos por los cuales el consumidor deja de utilizar los productos adquiridos. Comenzando con el análisis de la primera pregunta (anexo II), queremos señalar que en su elaboración se puso un especial interés en conocer el «tiempo real» (medido en horas y en minutos) que dedican los entrevistados a una actividad implícita en el acto de compra como es el "ver escaparates». Muy pronto se captó lo difícil que puede ser para cualquier persona cuantificar el número de minutos diarios que dedica a ver escaparates, puesto que en numerosas ocasiones es algo que se realiza casi sin tener conciencia de ello: Muchas personas, en sus desplazamientos habituales dentro del núcleo urbano (para ir al trabajo, asistir a espectáculos, etc.), y en ocasiones de forma inconsciente, "aprovechan" y van observando y "analizando" lo que se expone en los distintos establecimientos comerciales.

10. Utilizaremos para ello un estudio sobre hábitos de consumo realizado en Navarra en la primavera de 1993. Las características y la ficha técnica del estudio aparecen explicadas en el anexo I. 
Cuadro 1, pregunta 1. ¿Cuánto tiempo pasa usted a la semana viendo escaparates?

- Casi todos los días me detengo a ver escaparates, ya que así veo lo que necesito

- De vez en cuando, cuando veo algo que me llama la atención, pero son pocas veces

- Solamente me preocupo de ver escaparates cuando voy a comprar algo, o cuando necesito alguna cosa

— Nunca me detengo a ver escaparates

La pregunta 1, formulada definitivamente como se muestra en el cuadro 1 , cumple los objetivos previstos, ya que permite cuantificar tres tipos de personas que se detienen (con mayor o menor frecuencia) a conocer los productos expuestos en los escaparates. De la misma forma, hay una cuarta opción para aquellos que nunca ven escaparates. La opción «De vez en cuando, cuando veo algo que me llama la atención pero son pocas veces» debe interpretarse no en su sentido puramente literal, sino contextualizada dentro de las opciones disponibles en las que toma una "posición intermedia" entre un «detenerse a ver escaparates habitualmente» o hacerlo únicamente en condiciones de necesidad.

De este modo se ha llegado a conocer que el $37,3 \%$ de los entrevistados se detienen a ver escaparates con relativa frecuencia, y que un $17,8 \%$ nunca lo hacen. En el centro, en la frontera entre la necesidad real y la concepción subjetiva que cada uno tiene de sus necesidades, se encuentran un $44,7 \%$ de los entrevistados.

El resto de preguntas nos permitirán conocer las características de este colectivo que ve escaparates solamente cuando va a comprar algo o necesita alguna cosa, aunque antes de entrar en un estudio pormenorizado de éstas, se van a analizar los diferentes subgrupos formados por las variables clasificatorias:

El tipo de familia es uno de los factores que aporta más información a la hora de establecer diferencias en el tiempo transcurrido viendo escaparates; permitiendo caracterizar a las parejas sin hijos como el colectivo que más tiempo emplea en esta tarea, las familias (extensas y nucleares) por una frecuencia intermedia, y los que viven solos como los que menos tiempo emplean viendo escaparates.

El análisis de la edad desvela que los menores de 35 años se caracterizan por un elevado porcentaje de tiempo dedicado a ver escaparates, y por un escaso número de personas que nunca se detienen a verlos. En el extremo opuesto, el grupo de edad entre 46 y 55 años es el que ofrece los porcentajes más altos 
Cuadro 2. Tiempo empleado en ver escaparates y tipo de familia

\begin{tabular}{lllll}
\hline & $\begin{array}{l}\text { 2 núcleos } \\
\text { F. extensa }\end{array}$ & $\begin{array}{l}\text { Familia } \\
\text { nuclear }\end{array}$ & $\begin{array}{l}\text { Solo, piso } \\
\text { compartido }\end{array}$ & $\begin{array}{l}\text { Pareja } \\
\text { sin hijos }\end{array}$ \\
\hline Todos los días & 9,0 & 10,7 & 2,1 & 12,1 \\
De vez en cuando & 18,9 & 28,7 & 36,2 & 29,3 \\
Cuando voy a comprar & 53,2 & 41,8 & 34,0 & 1,7 \\
Nunca me detengo & 18,9 & 18,8 & 27,7 & 1,7 \\
\hline Total: & $\mathbf{1 1 1}$ & $\mathbf{3 8 3}$ & $\mathbf{4 7}$ & $\mathbf{5 8}$ \\
\hline
\end{tabular}

de "nunca me detengo a ver escaparates", y los más bajos en "Casi todos los días". Además de estas variables, también aportan diferencias importantes:

- El estado civil: El colectivo de los solteros es el que más tiempo dedica a ver escaparates: Un 48,2\% "Casi todos los días" y «Cuando ven algo que les llama la atención»; porcentaje que en los casados se reduce al $30 \%$. El $50 \%$ de los casados se detienen en los escaparates únicamente cuando van a comprar algo, dato que en los solteros se reduce al $37 \%$.

Ante la sospecha que esta relación pudiera estar encubriendo el influjo de una tercera variable - puesto que en otras investigaciones se ha constatado que el colectivo de solteros está compuesto, en su mayoría, por personas jóve-

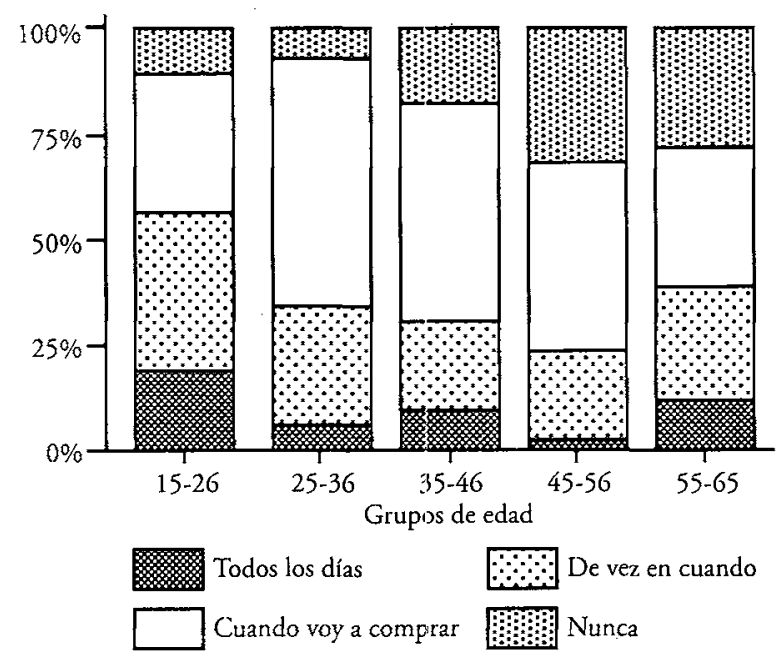

Gráfico 1. Frecuencia de ver escaparates en los distintos grupos de edad (en porcentajes). 
nes- se utilizó la técnica estadística de la neutralización de variables a fin de conocer las relaciones espúreas (no manifiestas) entre éstas. Mediante este procedimiento ha sido posible descubrir que una vez eliminado el influjo de la edad no hay asociación entre el tiempo empleado viendo escaparates y el estado civil o, dicho de otro modo, el factor que realmente produce estas diferencias es la edady no el estado civil.

- El sexo: $\mathrm{Al}$ analizar este comportamiento según el sexo parece ser que detenerse a ver los escaparates es más propio de mujeres que de hombres, puesto que un $30 \%$ de los hombres nunca se detienen a verlos, porcentajes que en las mujeres se reduce al $5,5 \%$.

- A medida que aumenta el nivel de ingresos se incrementa el número de las personas que ven escaparates habitualmente; "todos los días" $y$ "de vez en cuando". De modo que las personas con altos ingresos se caracterizan por detenerse frecuentemente a ver escaparates.

Señalar, no obstante, que otras variables como el tamaño del municipio en el que se reside y el nivel de estudios no presentan influencia significativa.

Una alta frecuencia de ver escaparates es, a fin de cuentas, un tiempo «invertido» en procesos de compra, inversión que puede materializarse o no en la realización de una compra. No obstante, el acto de "ver escaparates" es un elemento que sin duda alguna aumentará la probabilidad de realizar compras, independientemente que éstas sean necesarias o no. Podría enunciarse, a modo de subhipótesis o hipótesis parcial, que hay una gran relación entre el tiempo dedicado a "ver escaparates" y el número de compras innecesarias.

Ante la dificultad de definir operativamente lo que cada persona entiende por necesidad (Doyal y Gought, 1994: 73-74, 195-217), se considerará que comprar cosas que no se utilizan significa adquirir productos innecesarios ${ }^{11}$. Los datos mostrados en el cuadro 3, al indicar que únicamente algo menos de la mitad de los entrevistados "Nunca compran cosas que no usan", parecen convertirse en un "dato acusador», un hecho que cuestiona e invita a reflexionar sobre la "racionalidad" de nuestra conducta.

Como ya ha sido expuesto, una de las características del actual sistema productivo es que nos estimula constantemente, por medio de la publicidad y otros mecanismos comerciales, a "desear" y adquirir nuevos productos. Podría criticarse este planteamiento afirmando que las propias necesidades

11. Después de revisar la tipología propuesta por Bradshaw (necesidad normativa, percibida, expresada y comparada) consideramos que no es aplicable en este contexto debido, fundamentalmente, a la dificultad de establecer criterios objetivos para definir la «necesidad normativan, a la vez que no recoge las necesidades no conscientes: puede aparecer una necesidad no percibida que nos induzca a realizar una adquisición, en línea con una afirmación de $P$. Soler Pujals cuando señala que «un gran número de autores e investigadores del consumo indican en sus trabajos la importancia de las compras que realizan los consumidores apoyadas en una explicación de tipo emocional o subconsciente" (1990: 179). 
Cuadro 3, pregunta 3. ¿Suele comprar cosas que luego no usa? (Se hace referencia a ropa y calzado)

\begin{tabular}{lr}
\hline - Sí, frecuentemente & $8,3 \%$ \\
- Alguna que otra vez & $44,3 \%$ \\
- No, nunca & $47,2 \%$ \\
\hline
\end{tabular}

de los individuos han aumentado, ya que la gran mayoría de las personas viven en ciudades y realizan trabajos «limpios» - propios del sector terciario- en los que la apariencia externa es muy importante. Incluso admitiendo la premisa que las necesidades objetivas de las personas han aumentado, una compra racional debería caracterizarse por un uso, por un consumo de todos los productos comprados: «No se puede decir que, en general, los hábitos de compra de los españoles sean irracionales, [...] pero sí podemos hablar de hábitos poco o nada racionales en la medida en que esos usos se aparten de la adecuación de medios a fines que caracterizan a un consumidor prudente" (De Miguel, 1990: 69).

Diversas variables influyen de forma significativa en comprar productos que no se usan:

- El análisis del estado civil, mientras que algo más de la mitad de los casados nunca compran productos que no usan, este porcentaje se reduce al $36 \%$ en el caso de los solteros.

- La edad muestra como los menores de 25 años compran con más frecuencia productos que no utilizan, hábito que se reduce a medida que aumenta la edad: Un $25 \%$ de los menores de 25 años nunca compran productos que no utilizan, frente a un $61 \%$ del grupo entre $46-55$ años. En el grupo de más edad el porcentaje de compras de productos que no se utilizan vuelve a reducirse.

- Un 57\% de los hombres nunca compra productos que no utilizan, porcentaje que en las mujeres se reduce al $37 \%$.

En este sentido - tras observar la importancia del sexo en los diversos comportamientos analizados-, y pese a que en determinados ámbitos sociales se

Cuadro 4. Compra de productos que no se utilizan y edad

\begin{tabular}{llllll}
\hline & $\mathbf{1 6 - 2 5}$ & $\mathbf{2 6 - 3 5}$ & $\mathbf{3 6 - 4 5}$ & $\mathbf{4 6 - 5 5}$ & $\mathbf{5 6 - 6 5}$ \\
\hline Frecuentemente & 10,5 & 12,9 & 5,0 & 3,1 & 8,0 \\
Alguna vez & 64,3 & 39,3 & 40,8 & 35,4 & 36,0 \\
Nunca & 25,2 & $\mathbf{4 7 , 9}$ & 54,2 & $\mathbf{6 1 , 5}$ & 56,0 \\
\hline Total & $\mathbf{1 4 3}$ & $\mathbf{1 4 0}$ & $\mathbf{1 2 0}$ & $\mathbf{9 6}$ & $\mathbf{1 0 0}$ \\
\hline
\end{tabular}


está produciendo una "difuminación" de la línea divisoria entre los sexos ya que cada uno va asumiendo los papeles del otro (Andrés Orizo, 1979: 239 y 1992: 249; De Miguel 1993: 249); en el ámbito determinado del consumo el «sexo" sigue mostrando unas concepciones y hábitos de consumo diferenciados, tal y como manifiesta José Castillo (1991: 58-59) cuando señala que aunque el hombre se está incorporando a tareas caseras, esta incorporación se lleva a cabo muy lentamente, y sobre todo se incorpora no en tareas rutinarias sino cuando se cumplen determinadas circunstancias que dan a esas tareas un "rango distinto a la propia cotidianidad». Tal y como afirma este autor: «la rutinaria e innoble tarea de la mujer es enaltecida al rango de ocupación seria y digna por medio del concurso del varón» (1991: 59).

Por otro lado, hay una relación entre altos niveles de ingresos y compra frecuente de productos que no son utilizados: $\mathrm{El} 65,5 \%$ de los entrevistados con ingresos superiores a 275.000 pesetas compran «frecuentemente y alguna que otra vez» productos que no se utilizan.

Debe señalarse, no obstante, que no influye el hábitat o tamaño del municipio, el nivel de estudios ni el tipo de familia.

Desde otra perspectiva, el análisis de la pregunta 1 muestra una relación directa entre el tiempo empleado viendo escaparates y el número de productos que se compran y no son utilizados, ya que únicamente el $27 \%$ del colectivo que ve escaparates todos los días nunca compra productos que no utiliza. Por otro lado, el $61,7 \%$ de los que no se detienen a ven escaparates nunca compran productos que no utilizan.

No obstante, podrían «afinarse» más los resultados expuestos en la pregunta 3 si se volviera a preguntar ;cuanto? se utiliza cada producto, es decir, plantear el hecho de si hay muchos objetos que, utilizados unas pocas veces, se dejan en el armario condenados al olvido sin estar "físicamente desgastados». Este abandono prematuro no es consecuencia del «desgaste» producido por el uso continuado del producto, sino de un elemento mucho más "subjetivo" como es la obsolescencia producida por la evolución de la sociedad, tal y como ha sido definida anteriormente por Jean-Paul Ceron y Jean Bailon.

Cuadro 5 . ¿ Suele comprar cosas que después no llega a utilizar? y ¿̨ Cuánto tiempo pasa a la semana viendo escaparates?

\begin{tabular}{|c|c|c|c|c|}
\hline \multirow[b]{2}{*}{$\begin{array}{l}\text { Compra de } \\
\text { productos no utilizados }\end{array}$} & \multicolumn{4}{|c|}{ Ver escaparates } \\
\hline & $\begin{array}{l}\text { Todos } \\
\text { los días }\end{array}$ & $\begin{array}{l}\text { De vez } \\
\text { en cuando }\end{array}$ & $\begin{array}{l}\text { Cuando } \\
\text { compro }\end{array}$ & $\begin{array}{l}\text { Nunca me } \\
\text { detengo }\end{array}$ \\
\hline Frecuentemente & 20,3 & 8,5 & 6,4 & 6,5 \\
\hline Alguna vez & 52,5 & 58,2 & 39,3 & 31,8 \\
\hline Nunca & 27,1 & 33,3 & 54,3 & 61,7 \\
\hline Total & 59 & 165 & 267 & 107 \\
\hline
\end{tabular}


Cuadro 5, pregunta 2. Cuando deja de usar prendas, ¿̨suele ser porque están ya en mal estado, porque ya no le gustan o porque no están de moda?

$\begin{array}{ll}\text { Mal estado } & 52,6 \% \\ \text { No me gustan ya } & 32,3 \% \\ \text { Se han pasado de moda } & 15,1 \%\end{array}$

La pregunta 2 hace referencia a este hecho, cuestionando directamente cuales son los motivos por los cuales se dejan de utilizar las prendas: Algo más de la mitad de los entrevistados $(52,6 \%)$ deja de usarlas cuando están en mal estado, un $32,3 \%$ se atienen a criterios estéticos (no me gustan ya) y un mínimo $15,1 \%$ reconocen la influencia de la moda.

En el cuadro 6 pueden observarse los rasgos asociados a cada respuesta. Como en anteriores ocasiones, vuelve a aparecer la oposición entre jóvenes y que dejan de usar fundamentados en criterios de gusto, frente a edades superiores y nivel de estudios básicos, que tratan de aprovechar más las prendas y dejan de usarlas cuando se encuentran en mal estado.

"Comprar objetos que no se llegan a utilizar» (pregunta 3) presenta una gran relación con esta cuestión; ya que de los entrevistados que dejan de usar la ropa cuando ésta se encuentra en mal estado el $61 \%$ nunca compra productos que no utiliza, cifra que se reduce al $25,9 \%$ en los que dejan de usar ropa cuando no les gusta (cuadro 7 ).

Analizando la relación entre los motivos para dejar de utilizar prendas y el tiempo viendo escaparates (pregunta 1), se comprueba que las bajas frecuencias de ver escaparates están asociadas a dejar de usar prendas atendiendo a su mal estado. Por otro lado, el dejar de utilizarlas porque "ya no me gustan" es más frecuente entre las personas que más tiempo emplean viendo escaparates.

Una vez conocidos qué colectivos aportan diferencias más importantes en estos comportamientos será interesante continuar por esta línea investigado-

Cuadro 6. Rasgos caracteriologicos asociados a cada respuesta

\begin{tabular}{|c|c|c|c|}
\hline & Mal estado & No me gustan & Pasadas de moda \\
\hline Nivel de estudios & Básicos & Medios & Superiores \\
\hline Tipo de familia & Sólo & Sólo & Pareja sin hijos \\
\hline Estado civil & Casado & Soltero & Casado \\
\hline Edad & $36-55$ & $16-25$ & $46-55$ \\
\hline Ingresos & -100.000 & +275.000 & $200-275$ \\
\hline Sexo & Hombre & Hombre & Mujer \\
\hline
\end{tabular}


Cuadro 7. ¿Suele comprar cosas que después no llega a utilizar?; ¿Cuánto tiempo se pasa a la semana viendo escaparates? y ¿Por qué deja de usar prendas?

\begin{tabular}{llll}
\hline & \multicolumn{3}{l}{ Por qué deja de usar prendas } \\
\cline { 2 - 4 } & Mal estado & No gustan & Pasadas de moda \\
\hline Comprar no usar... & & & \\
Frecuentemente & 2,9 & 15,3 & 10,1 \\
Alguna vez & 35,8 & 58,7 & 46,1 \\
Nunca & 61,3 & 25,9 & 43,8 \\
Tiempo escaparates & & & \\
Todos los días & 5,8 & 17,5 & 9,0 \\
De vez en cuando & 22,6 & 36,5 & 25,8 \\
Cuando voy a comprar & 49,0 & 36,0 & 50,6 \\
Nunca me detengo & 22,6 & 10,1 & 14,6 \\
\hline Total & 310 & $\mathbf{1 8 9}$ & $\mathbf{8 9}$ \\
\hline
\end{tabular}

ra a fin de detectar subcolectivos o tipologías de sujetos que manifiesten opiniones contradictorias entre ellos. Con este fin utilizaremos el análisis tipológico categorial, cuyo fin es "buscar" comportamientos y mentalidades opuestos, sintetizar y mostrar hábitos contradictorios entre distintos tipos de sujetos. En este sentido, la combinación de estas tres variables permiten seleccionar diversos tipos de sujetos con comportamientos muy diferentes, tal y como se muestra en el cuadro 8.

Seleccionando las personas que van a ver escaparates "de vez en cuando" y dejan de usar la ropa cuando se ha pasado de moda y no les gusta (92 personas, un $15,3 \%$ de la población), el $79 \%$ compra productos que después no utiliza. Este porcentaje se reduce al $30 \%$ al analizar estas compras en el colectivo que nunca va a ver escaparates y que deja de usar la ropa cuando se encuentra en mal estado.

Cuadro 8. Análisis tipológico categorial preguntas 2, 1 y 3

\begin{tabular}{|c|c|c|c|c|c|c|}
\hline \multirow{2}{*}{$\begin{array}{l}\text { Pregunta } 2 \\
\text { Deja de usar } \\
\text { atendiendo al... }\end{array}$} & \multirow{2}{*}{$\begin{array}{l}\text { Pregunta } 1 \\
\text { Va a ver } \\
\text { escaparates }\end{array}$} & \multirow{2}{*}{$\begin{array}{l}\text { Pregunta } 3 \\
\text { Compra cosas } \\
\text { que no usa }\end{array}$} & \multicolumn{4}{|c|}{$\begin{array}{l}\text { Rasgos caracteriológicos } \\
\text { asociados a cada tipo }\end{array}$} \\
\hline & & & Sexo & Edad & E. civil & $\begin{array}{l}\text { Ingresos } \\
\text { pts/mes }\end{array}$ \\
\hline \multirow[t]{2}{*}{ Gusto y a la moda } & De vez en cuando & $79,3 \%$ & mujer & -25 & soliero & +200.000 \\
\hline & Todos los días & $73,2 \%$ & mujer & -35 & - & $150-275.000$ \\
\hline \multirow[t]{2}{*}{ Mal estado } & Cuando va a comprar & $33,6 \%$ & hombre & $36-55$ & - & +200.000 \\
\hline & Nunca & $30,0 \%$ & hombre & -45 & casado & -200.000 \\
\hline
\end{tabular}


Una de las grandes ventajas de esta técnica estadística es que muestra los rasgos caracteriológicos asociados a cada una de las tipologías encontradas, de modo que permite conocer detalladamente las personas que realizan cada una de estas conductas: Los colectivos que utilizan prácticamente todos los productos comprados se caracterizan por una elevada presencia de hombres, menores de 45 años, casados y con unos ingresos inferiores a las 200.000 pesetas mensuales. En el otro extremo de la clasificación, las compras frecuentes de productos que no se utilizan son frecuentes en las mujeres, los jóvenes menores de 35 años, los solteros y aquellos que residen en unidades familiares con ingresos superiores a las 200.000 pesetas mensuales.

\section{Reflexión final}

Estos resultados indican que el propio acto de compra - con todo el ritual que lleva implícito- es un elemento que reporta una gran satisfacción al comprador, y nos invitan a reflexionar sobre la importancia que los hábitos de compra tienen en la vida cotidiana de las personas. Si adoptamos un punto de vista más amplio, lo que estos resultados están sugiriendo es que el acto de compra está adquiriendo cada vez un papel más importante en la estructuración de la vida cotidiana de los individuos; de modo que podría formularse que esta acción es un elemento de integración social, tal y como ha sido planteado por Kevin Hetherington (1992: 97).

Este hecho, unido a la argumentación de numerosos autores que consideran que el trabajo está perdiendo la centralidad que antaño tenía en la vida de los hombres (Dahrendorf, 1979: 106, 304 y 1990: 298-300; Habermas, 1987: 513; Gortz, 1981 y 1994; Touraine, 1993: 130; Bocock, 1995: 33, 73, 114 y 120; etc.), nos conduce a enunciar — si bien tímidamente - que se está produciendo un desplazamiento de la sociedad centrada en el trabajo a la sociedad "sin centro" (Beriáin, 1990: 98), en la que el rol de consumidor emerge como una nueva forma de inclusión e integración social. No obstante, $y$ ante la rigurosidad de esta afirmación, procederemos a exponer con detalle los diferentes elementos que intervienen en la elaboración de este planteamiento.

En primer lugar, el trabajo "participa" cada vez menos en la vida de los individuos, no sólo porque se ha reducido el tiempo y la dedicación que el trabajador invierte en su lugar de trabajo, sino también debido a que se está produciendo una segmentación creciente dentro de los asalariados que "resquebraja” la propia concepción de los trabajadores como un "grupo unitario" (Giddens, 1979: 214). En esta línea, J.L. Santacoloma considera que la elevada importancia otorgada al trabajo en las sociedades actuales (un 93\% de los españoles lo consideran «muy y bastante importante»; Andrés Orizo, 1992: 42) debe interpretarse no en términos de "laboriosidad», sino de estricta necesidad, ya que «un puesto de trabajo es muy importante, pero no tanto el hecho de trabajar, considerado en sí mismo" (Santacoloma, 1992: 120). Estos aspectos nos llevan a afirmar que «la condición de trabajador, en cuanto tal, apenas resulta ya válida como punto de partida para llegar a agregaciones culturales, organizaciones y a interpretaciones colectivas» 
(Offe, 1992: 28) debido, fundamentalmente, a que otras funciones van ganando en importancia dentro de su vida (Inglehart, 1991: 227-266).

En segundo lugar, y a tenor de lo expuesto hasta el momento, consideramos que el rol de "consumidor" adquiere funciones de una nueva integración social, no sólo porque en el acto de compra demuestra una capacidad social previa, un "saber decidir" que demuestra mi aprendizaje social; sino fundamentalmente porque estas prácticas sociales se convierten en "comportamientos signo" con objeto de manifestar la pertenencia del individuo a grupos sociales que poseen más o menos "clase», más o menos "distinción-pretensión-necesidad" (Bourdieu, 1984: 28, 53-66 y 397-403). Estos aspectos muestran la doble naturaleza del consumo como integración y diferenciación según el modelo social que se desea imitar o de los cuales se quiere uno alejar o desmarcar (Pérez Tornero, 1992: 84).

En esta línea, y si recordamos los resultados obtenidos mediante el análisis tipológico categorial en el que se indicaba que los comportamientos más "consumistas" ${ }^{12}$ son propios de colectivos jóvenes, obtenemos una manifestación concreta de la importancia del rol de consumidor en las generaciones jóvenes; hecho que nos induce a cuestionar si es correcto definir la «importancia concedida al acto de compra" del colectivo joven como algo "coyuntural» -en la medida que se verá modificada con el aumento de la edad y la adquisición de obligaciones y compromisos que ello conlleva- o si por el contrario son conductas ya asentadas en la estructura de la personalidad de cada uno. Nuestro punto de vista es que estos comportamientos - adquiridos a través de un proceso de socialización enmarcado en un contexto social de abundancia- forman parte de la personalidad de cada uno y, si bien pueden cambiar en un momento dado por problemas coyunturales, volverán a su situación inicial una vez superados esos problemas (Inglehart, 1991: 9).

No obstante, y considerando otra perspectiva, otros autores analizan la «otra cara del consumo" y consideran que la creación y desarrollo de la sociedad de consumo es una nueva forma de alienación social al «desviar» la atención del plano político y la conquista de libertades a la elevación del nivel de vida (Navarro, 1991: 40; Rebollo Arévalo, 1978: 96; Touraine, 1993: 293). Para José Jimenez Blanco el propio concepto «sociedad de consumo» tiene una connotación ideológica, ya que enmascara la realidad al desplazar la crítica a las estructuras del consumo en lugar de centrarlas en las estructuras de la producción» (Jiménez Blanco, 1969: 85-118), argumentación compartida por otros autores como A. Pérez Agote (1978: 102) y J. Habermas: «La institucionalización del modo de decisión se vuelve alienado desde la perspectiva del votante, se desvía compensatoriamente hacia el papel de cliente, de la misma manera que la carga de la normalización del trabajo alienado se la compensa por la vía del papel de consumidor» (Habermas, 1987: 495).

12. Caracterizados por emplear mucho tiempo viendo escaparates, dejar de usar los productos atendiendo al gusto a la moda, y adquirir productos que no son utilizados. 
En línea con esta perspectiva crítica, en un reciente trabajo publicado por L.E. Alonso y F. Conde se analiza el papel de la sociedad de consumo como "proceso de aculturización": Estos autores ponen de manifiesto como el consumo fue un elemento utilizado por el gobierno franquista con objeto de desviar la atención de los verdaderos problemas sociales al mostrar la mejora en la "calidad de vida" producida por el incremento en los niveles de consumo (Alonso y Conde, 1994: 84, 91 y 212).

No deseamos «cerrar» el contenido de este artículo decantándonos por una posición o por otra, sino que nuestro objetivo es animar a los lectores interesados a seguir investigando en los efectos desencadenados por esta nueva formación social, en línea con una proposición realizada por F. Tezanos en 1986, la que afirmaba que ulas consecuencias que el desarrollo de la sociedad de consumo tiene en el orden sociopolítico no hayan sido descubiertas en su totalidad", aunque algunas investigaciones han puesto en relación la "dinámica consumista" con la apatía sociopolítica, con la mayor o menor «integración en el sistema», con el «conservadurismo político», etc. (Tezanos, 1986: 28-68).

\section{Anexo I: ficha técnica del estudio}

El tamaño muestral estadísticamente requerido para estudiar de forma representativa la población navarra entre 16 y 65 años de edad se ha establecido en 600 entrevistas, que en el supuesto $50 / 50$ tiene un error muestral máximo para datos globales de $\pm 4 \%$ a un nivel de confianza del $95 \%$.

El tipo de muestreo utilizado es aleatorio estratificado por afijación proporcional en virtud de la variable hábitat o tamaño del municipio (menos de 1.000 habitantes, de 1.000 a 5.000 habitantes, de 5.000 a 10.000 y más de 10.000 habitantes) y zona geográfica, (montaña, zona media, ribera y Pamplona y comarca) y corregido por cuotas de sexo (hombre y mujer), edad (menores de 25 años, entre 26-35 años, 36-45 años, 46-55 años y 56-65 años). En la designación de las calles, número, piso y mano en que se ha realizado la entrevista se utilizó el método de «Random Route», y en la elección concreta de los entrevistados en cada domicilio el "Método de Kish".

El trabajo de campo fue realizado entre mayo y junio de 1993 mediante encuesta personal en el hogar de cada entrevistado. La duración media de cada entrevista fue de 20 minutos, oscilando entre un mínimo de 18 minutos en Pamplona y un máximo de 25 minutos en los municipios menores de 1.000 habitantes.

\section{Anexo II: preguntas utilizadas}

01. ¿Cuánto tiempo pasa usted a la semana viendo escaparates?

- Casi todos los días me detengo a ver escaparates, ya que así veo lo que necesito

- De vez en cuando, cuando veo algo que me llama la atención, pero son pocas veces.

- Solamente me preocupo de ver escaparates cuando voy a comprar algo, o cuando necesito alguna cosa

- Nunca me detengo a ver escaparates 
02. Cuando deja de usar prendas, ¿suele ser porque están ya en mal estado, porque ya no le gustan o porque no están de moda?

- Mal estado

- No me gustan ya

- Se han pasado de moda

03. ¿Suele comprar cosas que luego no usa?

- Sí, frecuentemente

- Alguna que otra vez

- No, nunca

\section{Bibliografía}

Alonso, L.E.; Conde, F. (1994). Historia del consumo en España. Madrid: Debate. ALONSO, L.E. (1990). «Proceso de trabajo y objeto de consumo». Sociología del Trabajo, núm. 8, p. 59-80.

AlONSO, L.E. (1986). «La producción social de la necesidad». Economistas, núm.18, vol. 2, p. 26-31.

ALONSO, L.E. (1985). "Los orígenes del consumo de masas: el significado de una tranformación histórica». Estudios sobre consumo, núm. 6, Ministerio de Sanidad y Consumo, p. 11-19.

ANDRÉs ORIZO, F. (1982). Los nuevos valores de los españoles. Madrid: SM.

ANDRES ORIZO, F. (1979). Cambio sociocultural y comportamiento económico. Madrid: Centro de Investigaciones Sociológicas.

Atalli, J.; Guillaume, M. (1976). El Antieconómico. Barcelona. Labor.

BAllestero, E. (1982). "Sobre una Teoría no Utilitarista del Consumo, con aplicaciones a la demanda de alimentos. Revista de Estudios Agro-Sociales, núm. 121, p. 29 62.

BAUdrILlaRd, J. (1988). El sistema de los objetos. Madrid: Siglo XXI. (Primera edición en 1969.)

BAUdRILlaRD, J. (1971). Critica a la economía política del signo. Madrid: Siglo XXI.

BELL, D. (1987). Las contradicciones culturales del capitalismo. Madrid: Alianza Universidad.

BERIÁIN, J. (1990). "Trabajo, Democracia y Complejidad Social». AA.VV.: Cuestiones en Torno al Trabajo Social, Madrid: Editorial Popular.

BOCOCK, R. (1995). El consumo. Madrid: Talasa Ediciones.

Bourdieu, P. (1988). La distinción. Criterio y bases sociales del gusto. Madrid: Taurus Humanidades.

BradSAW, J. (1972). "The concept of social need». New Society, núm. 30.

CASARES RipOLL, J. (1994). "Influencia de las marcas y las formas comerciales en el consumidor". Revista de Occidente, núm. 162, p. 107-120.

Castillo Castillo, J. (1987): Sociedad de consumo a la española. Madrid: Eudema.

Castillo Castillo, J. (1991). "Consumo y Bienestar". En Vidal-Beneyto, J. (ed.); Beltrán, M. España a debate. Tomo II: La Sociedad. Madrid: Tecnos.

CERON, J.P.; BAILLON, J. (1980). La Sociedad de lo efimero. Madrid: Instituto de Estudios de la Administración Local.

CHERivs, A. (1980). "El trabajo y los valores: Cambios en las sociedades industriales». Revista Internacional de Ciencias Sociales, Vol. XXXII, núm. 3.

Clark, E. (1988). La publicidad y su poder. Barcelona: Planeta. 
DAVID ANTHONY, P. (1980). «El trabajo humano y la péerdida de significado». Revista Internacional de Ciencias Sociales, Vol. XXXII, núm. 3.

DAHRENDORF, R. (1990): El conflicto social moderno. Madrid: Mondadori.

DAHRENDORF, R. (1979). Las clases sociales y su conflicto en la sociedad industrial. Madrid: Rialp.

Doyal, L.; GOUGH, I. (1994). Teoria de las necesidades humanas. Barcelona: Icaria.

Eroski, (1990). «Endeudamiento». Suplemento núm. 18. Elorrio.

FRIEDMAN, G. (1977). La Crisis del Progreso. Barcelona: Laia.

Featherstone, M. (1991). Consumer Culture \& Postmodernism. Nueva York: Sage.

Galbraith, J.K. (1985). La Sociedad Opulenta. Barcelona: Planeta Agostini.

GALBRAITH, J.K. (1992). La cultura de la satisfacción. Barcelona: Ariel.

GIDDENS, A. (1979). La estructura de clases en las Sociedades Avanzadas. Madrid: Alianza Universidad.

GONZÁLEZ, B. (1981). «Necesidades sociales y deporte». En AA.VV. Políticas deportivas e Investigación Social. Gobierno de Navarra: Pamplona, p. 118-158.

GORZ, A. (1981). Adiós al proletariado. Barcelona: Libros del viejo Topo/Ed. 2001. GORZ, A. (1994). La metamorfosis del trabajo. Madrid: Sistema.

Habermas, J. (1987). Teoria de la Acción Comunicativa. Madrid: Taurus, tomo II. HalBWACHS, M. (1970). La classe ouvriere et les niveaux de vie. París: Gordon and Breach. Heller, A. (1978). Teoria de las necesidades en Marx. Barcelona: Península.

HETHERINGTON, K. (1992). "Stonehenge and its festival: Spaces of consumption". En SHIELDS, R. (ed.). Lifestyle Shopping; Londres: Routledge, p. 83-98.

INGLEHART, R. (1991). El cambio cultural en las sociedades industriales avanzadas. Madrid: CIS-Siglo XXI.

IACCOCA, L. (1985). Iaccoca. Autobiografia de un triunfador. Barcelona: Grijalbo.

JiMÉNEZ BLANCO, J. (1969). "Ideologías sobre la Sociedad de Consumo en España». En Mínguez, A. y otros. España: ¿Una Sociedad de Consumo? Madrid: Guadiana de Publicaciones.

Katona, G. (1968). La Sociedad de Consumo de Masas. Madrid: Rialp.

LAERMANS, R. (1994). «Aprendiendo a consumir: Los primeros Grandes Almacenes y la formación de la moderna cultura del consumo". Revista de Occidente, núm. 162, p. 121-144.

Loudon, D.L.; Della, A.J. (1993). Consumer Behavior. Londres: Mc.Graw-Hill.

MARCUSE, H. (1987). El hombre unidimensional. Barcelona: Ariel.

DE Miguel, A. (1993). La sociedad española 1992-93. Madrid: Alianza Editorial.

DE Miguel, A (1990). Los Españoles. Madrid: Ediciones Temas de Hoy.

Montañes, F. (1991). "Menudo Cacao». El Mundo, 7 de diciembre.

NAVARRO, V. (1991). "Producción y Estado de Bienestar. El contexto político de las reformas». Sistema, núm. 102.

NAREDO, J.M. (1981). «El fetichismo del consumo». Transición. núm. 28, enero, p. 17-22.

Nussbaum, F. (1933). A History of the Economic Institutions of Modern Europe: An Introduction to Der Moderne Kapitalismus of Werner Sombart. Nueva York: Crofts.

OFFE, C. (1992). "Es el trabajo una categoría sociológica clave?». Mercado de trabajo y crisis de crecimiento. Madrid: Alianza Universidad.

O'Shaughnessy, J. (1987). Why People Eiuy. Oxford: Oxford University Press.

PÉrez Agote, A. (1978). Hacia un estatuto teórico del consumo: Jean Baudrillard o la abstracción lógica de la forma". En Jiménez Blanco, J.; Moya, C. Teoría Sociológica Contemporánea. Madrid: Tecnos, p. 87-110. 
Pérez Tornero, J.M. y otros (1992). La seducción de la opulencia. Barcelona: Paidós. Piñuel, J.L. (1983). Producción, Publicidady Consumo. Vol. I, Madrid: Fundamentos. REBOLLO ARÉVALO, A. (1978). «La transformación del consumo privado en España 1958-1974». En VV.AA., La Sociedad de Consumo y su futuro, el caso de España. Madrid: Ministerio de Sanidad y Consumo, p. 73-118.

Rebollo Arévalo, A. (1983). Estructura del consumo en las economías domésticas en España. Madrid: Instituto Nacional de Consumo. Ministerio de Sanidad y Consumo. RIESMAN, D. (1981). La muchedumbre solitaria. Barcelona: Paidós.

SANTACOLOMA, J.F. (1992). "Trabajo y economía". En Elzo, J. y otros. ¿Somos los vascos diferentes? Euskalerria en la Encuesta Europea de Valores. Bilbao: Deiker-Universidad de Deusto.

Sauermann, P. (1983). Psicología del Mercado. Barcelona: Herder.

SEMPRINI, A. (1992). Le marketing de la marque. París: Les Éditions Liaisons.

SHIELDS, R. (1992). "Spaces for the subject of consumption». En SHIELDS, R. (ed.), Lifestyle Shopping. Londres: Routledge, p. 1-20.

Simmel, G. (1977). Filosofia del Dinero. Madrid. Instituto de Estudios Políticos.

Silverstone, R.; Hirsch, E. (1994). Consuming Technologies. Londres: Routledge. SMITH, A. (1985). La Riqueza de las Naciones. Barcelona: Orbis, cap. VIII, Libro $1^{\circ}$. Soler Pujals, P. (1990). La investigación motivacional en Márketing y Publicidad. Bilbao: Deusto.

Touraine, A. (1993). Crítica de la modernidad. Madrid: Temas de Hoy.

TEZANOS, J.F. (1986). "Transformaciones en la estructura social española». En HERNÁNDEZ, F.; MERCADÉ, F. Estructura Social y Cuestión Nacional. Barcelona: Ariel. Veblen, T. (1974). Teoría de la Clase Ociosa. México: F.C.E. (1 a edición en 1899). 